\title{
Yves Clot \& Dominique Lhuilier (Eds.) (2010). Travail et santé et Agir en clinique du travail
}

Toulouse : Editions Erès

Régis Ouvrier-Bonnaz

\section{(2) OpenEdition}

\section{Journals}

Édition électronique

URL : http://journals.openedition.org/activites/2537

DOI : 10.4000/activites. 2537

ISSN : 1765-2723

Éditeur

ARPACT - Association Recherches et Pratiques sur les ACTivités

\section{Référence électronique}

Régis Ouvrier-Bonnaz, "Yves Clot \& Dominique Lhuilier (Eds.) (2010). Travail et santé et Agir en clinique du travail », Activités [En ligne], 8-1 | avril 2011, mis en ligne le 15 avril 2011, consulté le 22 septembre 2020. URL : http://journals.openedition.org/activites/2537 ; DOI : https://doi.org/10.4000/activites. 2537 


\title{
Analyse d'ouvrage
}

\section{par Regis Ouvrier-Bonnaz}

\author{
Groupe de Recherche et d'Etude sur l'Histoire du Travail et de l'Orientation (GRESHTO) \\ Centre de Recherche sur le Travail et le Développement (CRTD) - CNAM \\ regis.ouvrier_bonnaz@cnam.fr
}

\section{Yves Clot \& Dominique Lhuilier (Eds.) (2010). Travail et santé et Agir en clinique du travail. Toulouse: Editions Erès.}

Les deux ouvrages dont nous rendons compte, «Travail et santé » et « Agir en clinique du travail », dirigés par Yves Clot et Dominique Lhuilier, professeurs à la chaire de psychologie du travail du Conservatoire National des Arts et Métiers, rassemblent les textes du colloque international «Clinique du travail » qui a réuni au CNAM les 30 et 31 Mai 2008 une quarantaine d'intervenants et plus de huit cents participants. "L'un reprend le problème de la signification entre subjectivité, santé et travail. L'autre s'attache aux transformations du travail et explore les moyens d'action en clinique $d u$ travail en les confrontant aux ressources et aux limites du savoir» (Clot \& Lhuilier, T\&S, p. 9) ${ }^{1}$. La présentation de ces deux ouvrages est l'occasion de mettre à l'épreuve l'objectif de cette publication qui vise à favoriser la poursuite des dialogues engagés à l'occasion de cette manifestation.

Quinze années après la parution des livres de Rifkin (1996) et de Méda (1996) définissant le travail comme un fait social en voie de marginalisation, l'intérêt majeur des contributions des différents intervenants, psychologues - sociologues, ergonomes, économistes, philosophes, anthropologues, statisticiens - est de replacer le travail au centre de la vie sociale et individuelle. A la lecture de ces deux ouvrages, un premier constat s'impose : les transformations observées dans le monde du travail, loin d'enlever au travail sa centralité, en changent cependant les formes, dans un sens qui peut conduire, comme tendent à le montrer plusieurs intervenants, à un accroissement des contraintes et des violences subies par les salariés. Pour la sociologue Danièle Linhart, les modalités de modernisation du travail liées aux contraintes du marché aboutissent le plus souvent à l'arracher à la société et aux enjeux qui la traversent pour en faire une affaire d'entreprise et d'individu. Dans cette logique d'individualisation des pratiques de travail et de leur contrôle quasi exclusif par l'entreprise à travers les stratégies managériales, "une fragilisation de la subjectivité au travail apparaît et se développe au fur et à mesure que son cadre collectif s'amenuise » (T\&S, p. 118). Les différents auteurs s'efforcent ainsi, chacun à leur niveau, au plus près de leur appartenance disciplinaire ou professionnelle, de produire des cadres de pensée et d'action favorisant le traitement des questions sociales liées au travail et à son évolution. Clot et Lhuilier, dès l'introduction, précisent les contours de la clinique du travail. Elle "n'est pas une école, une théorie, mais elle pourrait être, si on prend soin à son développement, une ressource et un instrument pour l'action en milieu de travail » (ACT, p. 7). Dejours va dans le même sens quand il précise que "la clinique du travail n'est pas seulement une théorie, c'est aussi une praxie. Son action, la pratique clinique sur le terrain, a des incidences sur l'évolution de la condition humaine et sur les contraintes de travail » (T\&S, p. 128). L'intention de la clinique est d'emblée affirmée : "la clinique est action de transformation. Elle est aussi production de connaissances. Il s'agit alors de voir " comment s'articulent, se différencient, s'alimentent réciproquement ces deux projets en acte : l'action et le savoir, l'intervention et l'élaboration de connaissances » (Clot \& Lhuilier, ACT, p. 8). Les relations entre savoir et action sont précisément l'objet du texte de Katia Kostulski. Pour elle, "intervention et production de connaissances convoquent des cadres, des contraintes, des ressources très différents, voire peu compatibles simultanément » (ACT, p. 27). S'appuyant sur le cadre de la clinique de l'activité, elle montre à partir d'exemples puisés dans son

1. Dorénavant dans le texte, nous utiliserons pour indiquer les références, T\&S pour le livre « Travail et santé » et ACT pour « Agir en clinique du travail». 
activité d'intervenante et de chercheuse que l'action dans l'une ou l'autre des situations en changeant de destinataires, d'objet et d'horizon change de nature. Elle tente alors de différencier recherche et expertise à partir de la notion de métier qu'elle distingue de celle de collectif pour la placer au centre de ses interventions. "Si les professionnels souffrent aujourd'hui au travail, c'est pour une large part parce qu'ils perdent leur capacité à agir sur lui » (idem, p. 30). Entre l'action et la production de connaissances, Kostulski identifie un « entre deux » qui est celui de «savoirs co-produits », sortes «d'hybrides dialogiques », rencontre des concepts de métier et des concepts scientifiques. Cette posture épistémologique qui fait des professionnels les meilleurs connaisseurs de leur métier, inscrit les « hybrides dialogiques » du côté de l'intervention. On pense ici aux concepts potentiels de Vygotski (1934/1997), produits de la «collision » entre les concepts scientifiques et quotidiens, «formations hybrides » selon Bernard Prot qui, par leur nature même, jouent un rôle déterminant dans l'accès aux connaissances au travail. Pour ces deux auteurs, «le concept potentiel est le résultat d'une opération de sélection d'un trait significatif du concept quotidien à partir de la signification du concept scientifique » (Kostulski \& Prot, 2004, p. 428), les concepts potentiels se constituant quand les concepts scientifiques « ouvrent la porte à la prise de conscience ».

Dans un texte au titre évocateur «L'ignorance du clinicien », Prot définit, en s'appuyant sur l'analyse des rapports entre ces différents concepts, la clinique comme une activité de partage de préoccupations : "les connaissances du travail restent le fruit d'une recherche, inquiète parfois, insatisfaite toujours pour développer son pouvoir d'agir sur les choses, parmi les autres, avec et contre eux » (ACT, p. 123). Il considère les questions d'orientation professionnelle contemporaines au carrefour des milieux d'apprentissage, des milieux scolaires et des milieux de travail pour les aborder à partir d'un point de vue théorique sur l'abstraction, appréhendée comme une activité d'élargissement et de développement des concepts quotidiens. Pour les professionnels, il s'agit alors de : «s'abstraire d'un milieu pour mieux transformer l'expérience qu'on y a vécue en connaissance, et en disposer pour moderniser les milieux professionnels sans opposer connaissance et initiative » (idem, p. 123). L'initiative est ici considérée d'abord comme une déformation de ce qui est fait susceptible de déplacer les lignes du genre professionnel permettant à certaines conditions la reprise d'un travail collectif d'élaboration à propos du métier.

Les problèmes concernant la production de connaissances sur le travail traités dans les différents textes sont complexes. S'ils adoptent des formes parfois différentes, ils ne sont pas nouveaux. Comme le suggère Lhuilier, pour mener à bien le projet de différenciation de l'intervention et de la recherche, il faut être attentif aux travaux existants et «éviter de croire et de faire croire que l'on découvre ce qui en fait a déjà été montré » (ACT, p. 206). En accord avec cette mise en garde, Clot et Lhuilier rappellent les apports majeurs à la clinique du travail de deux psychiatres, parmi d'autres - Le Guillant (2006) et Tosquelles (2009) - dont plusieurs textes viennent d'être réédités chez Erès. Ce rappel qui s'inscrit dans la cohérence du travail de Billiard (2001) concernant l'étude de la naissance et du développement de la psychopathologie du travail en France, nous incite à ne pas oublier ce que nous devons aux « anciens ». Michèle Salmona (ACT, pp.185-202) rappelle par exemple l'importance de l'apport de Jean-Pierre Vernant concernant l'étude de la construction des savoirs et savoir-faire en lien avec l'action dans la Grèce antique. L'héritage des anciens garde le plus souvent une grande pertinence pour comprendre que les problèmes rencontrés aujourd'hui sont souvent d'anciens problèmes non résolus ou mal traités. Marc Bloch, le co-fondateur de l'école historique des Annales, ne disait pas autre chose quand il affirmait que "l'ignorance du passé ne se borne pas à nuire à la connaissance du présent ; elle compromet, dans le présent, l'action même » (1941/1974, p. 45). Si on est attentif à cette mise en garde, un effort important est à faire, concernant les problèmes rencontrés dans le monde du travail, pour éviter que les bonnes intentions ne deviennent de «nouvelles mauvaises solutions ». Pour Guillaume Le Blanc, cet effort est d'autant plus important que « contrairement aux apparences qui suggèrent une grande variété et une grande diffusion des formes de la clinique (il suffit de songer à la multiplication des cellules d'écoute dans tous les lieux professionnels mais aussi la célérité avec laquelle se mettent en place des dispositifs psychologiques 
d'aide aux victimes en tous genres), rien n'est moins évident que la clinique » (T\&S, p. 18).

Pour ce philosophe, la clinique est « un fait normatif » à la jonction de quatre éléments à articuler : des situations vécues, des conditions sociales données, des dispositifs institutionnels et des formes démocratiques. "C'est seulement si elle prend en compte comme objet d'élucidation des souffrances sociales engendrées par les formes actives de vulnérabilisation sociale des vies à l'intérieur d'un dispositif institutionnel soutenu démocratiquement que [la clinique] peut exister pleinement. La trajectoire clinique vaut non comme un désir de changer le désir des sujets en les adaptant aux symptômes dont ils souffrent; elle se développe comme un traitement critique de l'emprise des normes sociales sur les sujets malmenés par elles ». Du coup, «c'est bien du sujet vivant qu'il faut partir, c'est-à-dire de sa capacité à donner sens aux normes (idem, pp. 18-19), la relativité du normal dépendant en grande partie de la multiplicité des valeurs. Friedmann l'avait bien analysé dans sa critique du taylorisme, "par ce canal de la valeur tout un flot de réalités psychiques, morales et sociales fait irruption » (1946, p. 355). Dès lors, le caractère impersonnel et normatif des obligations au travail ne vaut que s'il permet de poser le sujet, simultanément, comme conscience initiale de soi mais aussi comme vivant social. Ce qui est en jeu, c'est la possibilité pour le professionnel d'agir seul ou avec d'autres sur son milieu de travail, l'activité au travail devenant alors un opérateur de santé. La référence à l'œuvre de Canguilhem est ici évidente. L'occasion nous est ainsi offerte de lire ou relire la remarquable analyse que Canguilhem (1947) fait du livre de Georges Friedmann «Problèmes humains du machinisme industriel ». Pour lui, la résistance du travailleur aux « mesures qui lui sont imposées du dehors doivent donc être comprises autant comme des réactions de défense biologique que comme des réactions de défense sociale et dans les deux cas comme des réactions de santé ». Il est donc important que "les ouvriers saisissent le sens de leur travail et se situent eux-mêmes au sein du nouveau milieu, c'est-à-dire en fin de compte qu'ils se réfèrent à eux-mêmes le milieu en même temps qu'ils se soumettent à ses exigences». Dans ces conditions, «l'ouvrier cesse de se sentir objet dans un milieu de contrainte pour s'apercevoir sujet dans un milieu d'organisation» (Canguilhem, 1947, p. 129). Fabienne Hanique aborde la question de la norme pour interroger la notion de sens dont elle dénonce l'utilisation trop rarement problématisée. Pour elle, «s'interroger sur la nature du sens dont on déplore la perte revient à se questionner sur le statut de la norme mise en échec » (ACT, p. 144). Dans cette perspective, elle identifie trois niveaux. Le premier niveau renvoie à la dimension technique ou opérative de la norme. Le second correspondant à la dimension éthique est proche de la notion de valeur. Lorsque cette dimension éthique est bousculée, «ce sont alors les registres de la morale et du politique qui viennent à être interrogés » (idem, p. 147). Dès lors, pour le clinicien, "travailler sur les questions de sens au travail l'expose donc à l'appréhender dans ses aspects techniques, éthiques, idéologiques, mais également politiques » (idem, p. 155).

Quelle que soit la nature de la référence disciplinaire mobilisée et de l'approche théorique travaillée, le concept d'activité est mis à l'épreuve par plusieurs auteur(e) $\mathrm{s}^{2}$. Un accord de principe structure leur argumentation : l'activité ne se réduit pas au registre de l'interpersonnel, au rapport entre deux ou plusieurs individus, «ce qui suppose l'inscription mais aussi la contribution à un espace transsubjectif qui dépasse l'activité de chacun »(Clot \& Lhuilier, T\&S, p. 10). "Autrement dit, l'activité relie aux autres et au monde physique, mais le soi est aussi un des destinataires de l'activité. Pas seulement pour se préserver, se conserver, mais aussi se développer »(Lhuilier, ACT, p. 207). Le développement devient alors une affaire individuelle mais aussi collective : "Le travailler, ou ce que l'on conviendra de désigner comme "le travail vivant" pour reprendre encore un terme de Marx, passe par la formation d'habiletés individuelles, et on le verra aussi, par la formation de compétences collectives qui doivent bel et bien être inventées, découvertes et appropriées par des travailleurs ensemble et par chaque travailleur lui-même » (Dejours, T\&S, p. 135). Si les intentions convergent, les visées de la clinique du travail peuvent être divergentes comme s'efforce de le montrer Thomas Périlleux dans sa comparaison des fondements de la psychodynamique du travail et de la clinique de l'activité pour étudier la montée en puissance de « la prescription subjective »- «la contrainte

2. On peut regretter ici que certain(e)s auteur(e)s ne soient pas suffisamment explicites sur le cadre théorique mobilisé. 
d'avoir à faire ses preuves en permanence, l'obligation de mettre au travail ses propres affects et valeurs pour atteindre les buts prescrits, l'intensification de la présence et de l'attention au travail » (T\&S, p. 61). Pour Gilles Amado (T\&S, pp. 65-78), élargissant la comparaison à d'autres approches, les démarches mises en œuvre en clinique du travail courent le risque de s'adresser à une partie seulement de la subjectivité, celle qui correspond avant tout au projet scientifique, pragmatique, politique du chercheur et de l'intervenant.

Au-delà de ces mises en garde, ce qui est en jeu, c'est le rapport entre activité et travail dont la nature contrairement aux apparences ne s'impose pas directement. Dans son approche thérapeutique en psychiatrie, Tosquelles (1972/2009) avait déjà attiré l'attention sur le piège que la notion d'activité pouvait cacher, « ce qui était connoté dans la notion d'activité -(...)- s'opposait d'une façon radicale à la simple bougeotte et même au mouvement entrepris, imposé ou proposé par l'autre que soimême » (2009, p. 25). Pour Clot, "si l'activité mérite d'entrer dans la clinique, c'est qu'elle peut devenir un opérateur pour le renouvellement du problème de la subjectivité. C'est qu'elle est finalement un quasi-synonyme de santé (...). Entrée dans la clinique, l'activité entraîne donc avec elle la liaison entre sujet et collectif. Elle est leur trait d'union. C'est seulement en elle qu'ils montent leurs rapports, et jusque dans sa paralysie (ACT, pp. 21-22). Ainsi, l'activité ne se réduit pas à un objet de savoir, elle est avant tout un moyen de vivre et de se développer avec les autres. "Vivre, c'est pouvoir développer les objets, destinataires et les instruments de son activité en affectant le monde par son initiative » (idem, p. 16). Dès lors, rien n'est jamais donné d'avance et aborder la clinique par la notion d'activité a des effets et des conséquences parfois difficiles à prévoir. En appui sur Tosquelles mais aussi sur l'approche historico-culturelle en psychologie de l'école russe, Clot le dit à sa façon, l'activité, "n'est rien d'autre que la conversion par les hommes du "milieu" en "monde". L'homme ne s'adapte pas; ou plutôt, il ne s'adapte qu'en adaptant le monde autour de lui pour le faire sien. Sans cette possibilité de travailler à construire son monde avec les autres hommes dans lequel il se "fera homme", il ne s'humanise pas» (idem, p. 15). A l'opposé d'une psychologie positiviste qui continue de penser l'Homme et son développement comme une unité abstraite, cette approche de l'activité nous invite à trouver dans "l'hominisation », à travers l'activité laborieuse, l'avènement de l'histoire sociale de l'humanité. Dans cette perspective, les outils relient les hommes à leur histoire. L'homme ne s'approprie pas le patrimoine social tel quel, entre l'individu et celui-ci, la division du travail multiplie les médiations. A côté du travail prescrit et du travail effectivement réalisé, Pascal Béguin identifie " tout un travail intermédiaire, nécessairement situé au sein d'un collectif, et dont la finalité est de transformer l'organisation officielle pour en faire un instrument effectif de l'action » (ACT, p. 130). Ce constat, nourri et enrichi de ses interventions et recherches sur la conception, l'amène à s'interroger pour savoir si l'organisation peut être considérée comme un instrument sous deux aspects : humain et artefactuel. Il montre que si la face humaine de l'organisation s'impose assez facilement, sa dimension artefactuelle est plus difficile à cerner comme tend également à le montrer Livia Scheller à propos de l'organisation du travail des conducteurs et conductrices de bus (T\&S, pp. 221-231). Les professionnels sont les mieux placés pour soigner l'organisation qui, en retour, leur fournir «l'énergie » nécessaire à l'action collective. Dans cette dynamique de réciprocité, les travailleurs réussissent à préserver leur santé quand ils trouvent les moyens de se reconnaître dans l'organisation officielle du travail pour en faire ensemble la source du développement de leur pouvoir d'agir.

Dans la cohérence de la genèse instrumentale, Jean-Luc Tomas montre, en prenant l'exemple de l'activité de chirurgiens, qu'il existe un genre corporel qui conserve les traces de l'histoire singulière des actions du sujet et de leurs conflits (ACT, pp. 279-294). On pense ici, bien évidemment à Mauss (1936) pour qui "le corps est le premier et le plus naturel instrument de l'homme. Ou plus exactement ... le premier et le plus naturel objet technique de l'homme, et en même temps moyen technique »(1936/1960, p. 372). En pensant de manière dialectique les rapports du biologique et du social, Mauss montre que le fonctionnement et le développement des techniques corporelles sont liés à des contextes sociaux-historiques précis mettant à disposition de chacun un ensemble d'outils 
culturels et techniques mis en œuvre dans des contextes intersubjectifs facilitant leur utilisation. Le processus d'appropriation et de développement des techniques du corps - ce que Seiris (1994,p. 128) commentant le texte de Mauss appelle la «technicisation des corps »- ne saurait être considéré en soi, déconnecté de ce qui lui donne son sens : l'activité. Sans cette technicisation, c'est-à-dire sans insertion active des corps dans le système des normes techniques vues comme source et ressources de l'activité individuelle et collective, aucune technique ne pourrait avoir prise sur le monde. Gabriel Fernandez, médecin et psychologue du travail montre de son côté comment les émotions vécues en situation de travail en se reproduisant dans la consultation médicale deviennent des instruments de développement autorisant ainsi « le développement concomitant des significations ». "Ces développements peuvent aider le sujet à remettre en question, lorsqu'ils existent, les buts fictifs qui dévoient son activité, que ces buts soient cognitifs ou affectifs. Ceci n'est réalisable qu'en prenant sur ce que font ses collègues ou sur ce qu'on a appris de leur travail, et c'est souvent un point de vue éloigné de celui du patient qu'il faut faire vivre, non pas pour trouver un illusoire juste milieu mais afin qu'il devienne pour le sujet un moyen de reconstruire du collectif en lui-même » (T\&S, p. 150). Dans son intervention, le médecin doit s'efforcer de comprendre comment l'expérience émotionnelle de la situation par le professionnel se forme pour l'aider à se servir du système émotionnel comme une ressource. Cette question des émotions et de leur instrumentalisation est également posée par le sociologue Marc Loriol à partir de l'analyse du travail de diplomates. La réponse apportée, en se situant du côté de la maîtrise du relationnel, apparaît alors comme étant très différente. Dans le cadre du travail diplomatique, l'émotion semble bien être cultivée comme une pratique ritualisée susceptible de jouer comme un code dès lors qu'elle est maîtrisée. L'émotion peut ainsi «être un outil de travail pour faire passer un message, obtenir la confiance ou gagner des avantages » (T\&S, p. 37). Dans une perspective proche, Marie-Anne Dujarier, propose de considérer la recherche-action clinique comme une démarche inverse de celle du management contemporain lorsqu'il promet de débarrasser le sujet de ses émotions encombrantes au travail (ACT, p. 87). Pour elle, la bataille du réel est aussi linguistique dans la mesure où "le management idéaliste utilise à son profit des mots issus de la critique sociale qui lui est adressée pour leur donner d'autres significations » (idem, p.89) et ainsi justifier l'intensification du travail et la flexibilité de l'emploi en éloignant progressivement le signifié du signifiant. Elle rappelle alors que les organisations comme les discours sont des constructions sociales reposant sur des rapports de force bien analysés dans le texte de Bernard Doray d'un point de vue psychanalytique sous l'angle de la marchandisation du travail (T\&S, p.25-36). Dans ce contexte, comme le rappelle Florence Osty traitant du problème de la reconnaissance au travail, « il s'agit tout d'abord de préserver la dynamique sociale du métier en prenant soin du travail (...). Prendre soin du métier suppose de prendre à bras le corps la question de la qualité du travail et celle de son évaluation comme centrales et objets d'un compromis productif à restaurer » (ACT, p. 277), l'intervention devant permettre aux professionnels de "quitter la scène de l'arbitrage solitaire des dilemmes pour un espace social de régulation » (idem, pp. 277-278).

On l'aura compris, rendre compte de la richesse de l'apport des différentes interventions dans un colloque dont la nature interdisciplinaire est affirmée, ne peut se faire sans la recherche des cohérences et des liens tissés entre les intervenants mais aussi en n'occultant pas les différences entre eux - ces dernières pouvant être une ressource pour les discussions à venir. Combiner souci d'exhaustivité et maîtrise du risque de dispersion n'est pas une tâche aisée. C'est pourquoi, à partir de quelques contributions, nous avons tenté de poser et discuter les contours conceptuels délimitant les questionnements mis en tension entre intervenants.

La répartition des textes telle qu'elle a été retenue par les deux coordonateurs en deux livres organisés respectivement en deux et trois chapitres peut faciliter la lecture. Pour notre part, nous avons organisé notre lecture autour de deux axes. Le premier concerne la place du sujet ou, plus précisément encore, la place de la subjectivité dans la clinique du travail - ce problème pouvant être abordé sous l'angle de la désubjectivation au travail comme dans le texte d'Eric Hamraoui (T\&S, pp. 101114). Le deuxième axe concerne la place respective du métier et du collectif dans la clinique du tra- 
vail traitée, à différents niveaux et de manière différente, par plusieurs intervenants, parfois comme outils de développement parfois comme éléments de régulation. Le rapport entre santé et travail est alors abordé du côté de l'activité comme opérateur de santé - l'activité pouvant être vue sous l'angle d'activités réflexives comme dans les textes de Damien Cru (ACT, pp. 67-82), de Malika Litim (ACT, pp. 95-106) ou de Jean-Pierre Minary (ACT, pp. 39-50) ou sous l'angle des ressources plus strictement dialogiques comme dans les textes de Maryse Bournel-Bosson (ACT, pp. 225-236) ou de Daniel Faïta (T\&S, pp. 79-100). Dans tous les cas, l'ancrage disciplinaire doit être dépassé pour ne pas enfermer la lecture dans une approche trop réductrice. Ainsi, le texte de Serge Volkoff et AnneFrançoise Molinié, «Quantifier sans broyer ? Les statistiques en santé au travail à la rencontre des analyses cliniques », n'est pas à lire sous le seul angle quantitatif mais aussi de manière qualitative du côté de la compréhension de l'activité des professionnels et de son évolution (T\&S, pp. 175-188). Pour être efficace, la justification du choix d'approche des textes est à faire par le lecteur lui-même. En procédant comme nous l'avons fait, nous espérons à travers nos propres tâtonnements et cheminements, encourager le lecteur à (re)trouver pour lui, à sa manière, les cohérences et les différences qui structurent les textes présentés dans les deux ouvrages pour en faire quelque chose à son niveau et ainsi prolonger l'œuvre engagée en les confrontant à d'autres sources et références théoriques. Pour conclure, si l'on suit Clot quand il précise que «la seule bonne pratique en clinique de l'activité est peut-être la pratique de la controverse professionnelle entre connaisseurs » (ACT, p. 24), il nous reste à souhaiter que la lecture de ces textes entretienne les divergences et les disputes entre tous les professionnels - chercheurs et praticiens - concernés par les thèmes développés, pour faire vivre et développer, à leur niveau, leur propre métier. La parution de ces deux ouvrages est l'occasion d'engager le débat.

\section{RÉFÉRENCES}

Billiard, I. (2001). Santé mental et travail. L'émergence de la psychopathologie du travail. Paris: La Dispute.

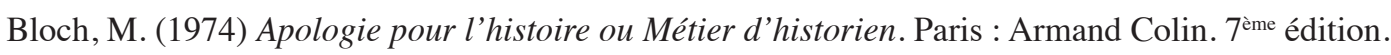

Canguilhem, G. (1947). Milieu et normes de l'Homme au travail. Cahiers internationaux de sociologie, III, 120-135.

Friedmann, G. (1946). Problèmes humains du machinisme industriel. Paris: Gallimard.

Kostulski, K., \& Prot, B. (2004). L'activité conversationnelle d'un jury de validation d'acquis : analyse interlocutoire de la formation d'un concept potentiel, Psychologie française, 49, 425-441.

Le Guillant, L. (2006). Le drame humain du travail. Essais de psychopathologie du travail. Toulouse: Editions Erès.

Mauss, M. (1936). Les techniques du corps, Journal de psychologie normale et pathologique, XXXII(3-4). Repris dans «Sociologie et anthropologie », Paris: PUF, 2ème édition, pp. 365-386.

Méda, D. (1996). Le travail, une valeur en voie de disparition. Paris: Aubier.

Rifkin, J. (1996). La fin du travail. Paris: La Découverte.

Seiris, J.-P. (1994). La technique. Paris: Gallimard.

Tosquelles, F. (2009). Le travail thérapeutique en psychiatrie. Toulouse: Editions Erès.

Vygotski, L. (1997). Pensée et langage. Paris: La Dispute.

Octobre 2010 